\title{
Antithrombotic Potential of Red Allium cepa and Angelica gigas Nakai
}

\author{
Jin Young Lee, Jae Seon Kang* \\ Department of Pharmacy, College of Pharmacy, Kyungsung University, Suyeong-ro, Nam-gu, Busan, KOREA.
}

\begin{abstract}
Introduction: Red Allium cepa and Angelica gigas Nakai is widely used in oriental medicine and also has various physiological activities. The main components of two substances will have potential benefits to blood flow improvement. Objectives: This study evaluated the cell viability and antioxidant effects of Red Allium cepa extract, Angelica gigas Nakai extract and their mixture and observed anticoagulation activity and antiplatelet aggregation activity. Methods: Red Allium cepa was bioconverted through the use of the Bacillus subtilis KJ-3 (BS3) strain after ethanol extraction (Red-BCQ). Angelica gigas Nakai extract (Agnex) contains a large amount of coumarins such as decursin and decursinol angelate (D/DA). Here, we observed antioxidant and antithrombotic effects after treatment with Red-BCQ, Agnex and mixture of these two compounds. Results: The quercetin content of Red-BCQ increased by about 7.4-fold after bioconversion. A $1 \mathrm{mg}$ portion of Agnex contained $0.4146 \mathrm{mg}$ of $\mathrm{D}$ and $0.3659 \mathrm{mg}$ of DA. Red-BCQ and Agnex exerted strong antioxidant activities due to their high polyphenol and flavonoid content. In our study, Red-BCO and Agnex exhibit beneficial effects to external coagulation pathways by significantly expanding aPTT. However, a combination of Red-BCQ and Agnex did not exert any synergistic effect on anti-coagulation activity. Additionally, our results concerning platelet aggregation inhibitory effects revealed that these effects increased by treatment with Agnex alone or in combination with Red-BCQ. Conclusion: Further research is required to clarify the underlying mechanism. However, Red-BCO and Agnex are considered to possess antioxidant effects either alone or as a mixture and also exert antithrombotic effects.
\end{abstract}

Key words: Red Allium cepa, Angelica gigas Nakai, Bacillus subtilis KJ-3, Bioconversion, Anti-coagulation.

\section{INTRODUCTION}

Eating a diet rich in vegetables and fruits is critical for reducing the risk of chronic degenerative diseases. ${ }^{1}$ It is established that vegetables and fruits contain antioxidant polyphenolic compounds such as flavonoids and phenolic acids that can remove free radicals. Flavonoids consist of several phenolic hydroxyl functional groups and the location and number of rings affect their reactivity. ${ }^{2-4}$ Onions (Allium cepa) contain a large amount of flavonoids and are one of the most important sources of polyphenols. Among onion flavonoids, quercetin and its glucosides contain $70 \%$ of the total flavonoids found within the onion. ${ }^{5}$ Other flavonoids that exist in small quantities are kaempferols, rutin and their derivatives, ${ }^{6-8}$ and red onions in particular have been reported to contain high levels of quercetin and high amounts of active ingredients such as anthocyanins that tend to increase consumption but possess short storage stability. Dang-gui is a useful plant resource that is a member of the family Umbeliferae. Angelica gigas Nakai is produced in Korea, Angelica acutiloba Kitagaw is produced in China and Angelica sinensis Diesel is produced in Japan and
Submission Date: 05-08-2020; Revision Date: 24-12-2020; Accepted Date: 03-05-2021

DOI: 10.5530/ijper.55.3.157 Correspondence:

Prof. Jae Seon Kang Dean of the College of Pharmacy Department of Pharmacy, Kyungsung University, 309, Suyeong-ro, Nam-gu, Busan, KOREA. Phone: +82-51-663-4882 Email id: jskang28@hanmail. net

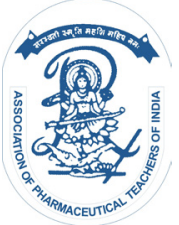

www.ijper.org 
members of the plant family are known to exert various pharmacological effects. They are particularly useful for the treatment of gynecological diseases and anemia and they can be used for sedation, analgesic effects, immune enhancement, antioxidant activities, arthritis treatment, anti-cancer treatment, alcohol metabolism promotion and liver disease improvement. ${ }^{9-14}$ The roots and stems of Agnex possess decursin, decusinol, decusinol angelate, nodakenetin, nodakenin, umbelliferone of the coumarine family, $\alpha$-pinene and $\beta$-sitosterol and the main components are decursin and decursinol angelate of the pyranocoumarin family. In Korean danggui (Cham-dang-gui), the component ratio of decursin and decusinol angelate is approximately 6:4. ${ }^{15}$ Kim et al. ${ }^{16}$ observed that decursin isolated from Chamdanggui could influence the inhibitory activity of ACAT enzymes controlling cholesterol absorption and release in the small intestine and liver, ultimately helping to prevent arteriosclerosis and hyperlipidemia. Blood is a vital component of many higher organisms, as it plays crucial roles in delivering oxygen, nutrients and metabolic waste through blood vessels and in controlling body temperature, moisture content, buffer capacity and constancy of the immune system. Blood loss from damaged blood vessels is prevented by the formation of thrombosis (blood clots) and the clot is degraded by a specific protease called plasmin. ${ }^{17}$ An abnormal increase in thrombotic formation in arteries and veins causes various thrombotic diseases such as myocardial infarction and ischemic stroke and this abnormal thrombotic formation is the main cause of a number of diseases and can result in death. ${ }^{18}$ Given this, a proper balance between thrombotic formation and coagulation is necessary. Thrombosis is a multifunctional disease that is induced by hyper coagulation, which is the formation or presence of a thrombus within a blood vessel. ${ }^{19}$ Thrombotic diseases such as heart disease and cerebrovascular thrombosis represent major causes of death and the incidence rate of these pathologies is increasing each year. ${ }^{20}$ Thrombosis is closely related to the adhesion, aggregation and secretion function of activated platelets and to the activation of intrinsic and extrinsic coagulation systems, all of which cause blood coagulation and fibrin formation. Although there are many causes, the accumulation of a thrombus is a key factor in the analysis of this disease. ${ }^{21}$ Based on this, our study aimed to investigate the antioxidant and antithrombotic effects of the quercetin component of red Allium cepa, the decursin and decursinol angelate (D/ DA) component of Angelica gigas Nakai and mixture of these compounds.

\section{MATERIALS AND METHODS Preparation of Red-BCQ powder}

The red Allium cepa was supplied by the Onion Research Institute of Agriculture and Technology (Gyeongsangnam-do, Changnyeong). A total of 16 $\mathrm{kg}$ of red Allium cepa (dried powder) was added into 80 liters of alcohol and stored for $3 \mathrm{hr}$ at $40^{\circ} \mathrm{C}$. This mixture was then filtered using the housing filter (pore size: $25 \mu \mathrm{m})$ and centrifuged $(10,000 \mathrm{rpm}, 15 \mathrm{~min})$ to obtain the supernatant. The concentrated supernatant is termed Red-Q and was used for biological conversion. The Bacillus subtilis KJ-3 (KCCM 11523P: BS3) strain was used for biological conversion. A single colony of the wild-type strain BS3 was inoculated into $5 \mathrm{ml}$ of TSB (tryptic soy broth) medium and then incubated overnight at $150 \mathrm{rpm}$ and $42^{\circ} \mathrm{C}$. Pre-cultured BS3 strains were grown at a $5 \%$ rate in mineral medium (corn starch $1.5 \mathrm{~g}$, yeast extract $0.5 \mathrm{~g}, \mathrm{~K}_{2} \mathrm{HPO}_{4} 0.25 \mathrm{~g}, \mathrm{KH}_{2} \mathrm{PO}_{4} 0.25$ g, $\mathrm{KCl} 0.05$ g, NaCl 0.03 g, $\mathrm{MgSO}_{4} 0.05$ g, $\mathrm{MnSO}_{4} 0.003$ $\mathrm{g}$ and $\mathrm{CaCl}_{2} 0.02 \mathrm{~g}$ ) with an incubation for $4 \mathrm{hr}$ at 150 $\mathrm{rpm}$ and $40^{\circ} \mathrm{C}$. The red-Q and culture medium were mixed at a $1: 4$ ratio, incubated at $55^{\circ} \mathrm{C}$ and $150 \mathrm{rpm}$ for $4 \mathrm{~h}$ and then stored at room temperature for $2 \mathrm{hr}$. After centrifugation for $15 \mathrm{~min}$ at $10,000 \mathrm{rpm}$, the sediment was dissolved in $70 \%$ ethanol. After a final step of centrifugation $(10,000 \mathrm{rpm}, 15 \mathrm{~min})$, the supernatant was concentrated and powdered by adding excipient to yield Red-BCQ powder. The Bacto ${ }^{\mathrm{TM}}$ tryptic soy broth used for strain culture was purchased from Becton Dikinson (Franklin Lakes, NJ, USA). The fermented cultivators used were Ecell3000s (Ecell), the shaking incubator used was Seyoung SH-803R (Seoul, Korea) and the centrifuge used LABOGENE centrifuges (scanspeed 1735R, Daejeon, Korea). HPLC (Waters 2690, San Diego, California, USA) was used to analyze the quercetin content of Red-Q, Red-BCQ, Red-BCQ powder. The calibration curve was prepared using the quercetin standard product and the specimen was filtered using a $0.45 \mu \mathrm{m}$ membrane filter to a concentration of $500 \mu \mathrm{g} / \mathrm{ml}$ and then analyzed. The analysis conditions are described below. Briefly, a Zorbax Eclipse XDB$\mathrm{C}_{18}$ column $(4.6 \mathrm{~mm} \times 250 \mathrm{~mm}, 5 \mu \mathrm{m})$ was used and the column temperature was $35^{\circ} \mathrm{C}$. The mobile phase consisted of $60: 40(\mathrm{v} / \mathrm{v})$ mixed with $2 \%$ acetic acid (A) and Acetonitrile (B). The flow rate was $1.3 \mathrm{~mL} / \mathrm{min}$, the injection amount was $20 \mu \mathrm{L}$, the detector used was photo diode array detector and the detection wavelength was $370 \mathrm{~nm}$.

\section{Preparation of Agnex powder}

Angelica gigas Nakai was purchased from Simmani Sansam Farming Association (Gyeongsang Nam-do Hamyang- 
Gun). A total of $15 \mathrm{~kg}$ of dried Angelica gigas Nakai powder was added into 75 liters of alcohol and stored for $3 \mathrm{hr}$ at $40^{\circ} \mathrm{C}$ prior to filtering with the mixture using the housing filter (pore size: $25 \mu \mathrm{m}$ ). The precipitate was obtained by adding a $2 \mathrm{X}$ volume of water to the filtrate, adjusting the $\mathrm{pH}$ to 5 and then centrifugation $(10,000 \mathrm{rpm}, 15 \mathrm{~min})$ after six hours. A $5 \mathrm{X}$ volume of $70 \%$ ethanol was added to the precipitate and the filtrate was concentrated to 20 Brix. The extract was powdered by adding excipient and the powdered form was termed Agnex (Angelica gigas Nakai extract) powder. The optimal conditions for decursin and decursinol angelate (D/ DA) detection were achieved using an HPLC (Agilent Technologies, 1100 series; U.S.A.), column (Agilent Zorbax SB-C $\left.{ }_{18}, 250 \mathrm{~mm} \times 4.6 \mathrm{~mm}, 5 \mu \mathrm{m}\right)$ and detection at $329 \mathrm{~nm}$ using a UV monitor (Agilent Technologies, 1100 series, Photo-diode Array UV/Vis detector; U.S.A.). The column was maintained at room temperature and the mobile phase consisted of $70: 30(\mathrm{v} / \mathrm{v} \%)$ acetonitrile and $0.1 \%$ formic acid.

\section{Polyphenol and Flavonoid Analysis}

The total polyphenol content was measured according to the Folin-denis method. ${ }^{22} \mathrm{~A}$ total of $500 \mu \mathrm{L}$ of FolinCiocalteu's phenol reagent was added to $500 \mu \mathrm{L}$ of the sample and then mixed. The mixture was allowed to react for six minutes at room temperature and a 500 $\mu \mathrm{L}$ volume of 10 percent $(\mathrm{w} / \mathrm{v}) \mathrm{Na}_{2} \mathrm{CO}_{3}$ solution was then added. The mixture was allowed to react for $1 \mathrm{hr}$ and the absorbance was subsequently measured at 725 $\mathrm{nm}$. The standard curve was constructed using the gallic acid as the standard. The total flavonoid content was measured using the Davis method. ${ }^{23}$ A total of $1 \mathrm{~mL}$ of diethylenglycol and $10 \mu \mathrm{L}$ of $1 \mathrm{~N} \mathrm{NaOH}$ were added to a $100 \mu \mathrm{L}$ volume of the sample, reacted for one hour at $37^{\circ} \mathrm{C}$ and then measured for absorbance at $420 \mathrm{~nm}$. The standard curve was constructed using naringin $(0.01 \mathrm{~g} /$ $\mathrm{mL})$.

\section{In vitro Antioxidant Activity}

DPPH (1,1-diphenyl-2-picryl hydrazyl) anion scavenging ability and nitrite scavenging activity were evaluated according to previously reported methods. ${ }^{24,25}$ Ascorbic acid (Sigma Co., St. Louis, MO, USA) was used as positive control. Each activity rating was expressed as the mean and the standard deviation of three replicates. DPPH radical scavenging activity was assessed according to the method described by Blois et al. ${ }^{24}$ A total of $0.15 \mathrm{mM}$ of DPPH solution (ethanol for control) was added to 50 $\mu \mathrm{L}$ of sample and reacted at room temperature for 30 min. After the reaction, the absorbance was measured at $517 \mathrm{~nm}$.
DPPH radical scavenging activity $(\%)=[1-\{(\mathrm{S}-\mathrm{SB}) /$ $(\mathrm{C}-\mathrm{CB})\}] \times 100$

S: Absorbance of sample, SB: Absorbance of sample blank, C: Absorbance of ethanol, CB: Absorbance of ethanol blank

The nitrite scavenging activity was assessed according to Griess reaction, ${ }^{25}$ with some modifications. RAW 264.7 macrophages were inoculated into 96-well plates and then pre-treated with each sample $(125$ and $1000 \mu \mathrm{g} /$ $\mathrm{mL}$ ) for $2 \mathrm{~h}$. The cells were subsequently stimulated with LPS $(200 \mathrm{ng} / \mathrm{mL})$ for $24 \mathrm{~h}$. NO concentration in the medium was determined using Griess reagent at $540 \mathrm{~nm}$ on a microplate reader (MQX200, Bio-Tek, USA) to allow for the calculation of the inhibition rate of NO.

\section{In vitro Anticoagulant Activity}

The samples were initially dissolved in dimethyl sulfoxide (DMSO) and the anti-coagulation activity was then assessed by measuring the clotting times required for activation of thrombin (T'T: thrombin time), prothrombin (PT: Prothrombin time) and coagulation factor (aPTT: active partial thromboplastin time) ${ }^{26} \mathrm{TT}$, PT and aPTT assays were conducted according to the manufacturer's protocols (Ameulungcoagulameter, Lemgo, Germany). Aspirin (1.5 mg/mL, acetylsalicylic acid, Sigma, St. Louis, MO, USA) was used as a positive control, as it played a role inhibiting thrombin generation and clot formation at high doses $(300-500 \mathrm{mg} / \mathrm{kg}) \cdot{ }^{27}$ All data are presented as the mean $\pm \mathrm{SD}$ values of triplicate experiments.

\section{In vitro Platelet Aggregation Activity}

The effects of the sample on platelet aggregation were measured using a Whole Blood Aggregometer (Chronolog, Havertown, PA, USA). ${ }^{28}$ The washed platelets (5 $\times 10^{8}$ cells $/ \mathrm{mL}$ ) were suspended in buffer containing $138 \mathrm{mM} \mathrm{NaCl}, 2.7 \mathrm{mM} \mathrm{KCl}, 12 \mathrm{mM} \mathrm{NaHCO}, 0.36$ $\mathrm{mM} \mathrm{NaH} \mathrm{PO}_{4}, 5.5 \mathrm{mM}$ glucose, $0.49 \mathrm{mM} \mathrm{MgCl}_{2}$ and $0.25 \%$ gelatin ( $\mathrm{pH} 7.4$ ). The samples were next added to the platelet and with the mixture was stimulated by the addition of $2.5 \mu \mathrm{L}$ of collagen $(1 \mathrm{mg} / \mathrm{mL})$ and then incubated at $37^{\circ} \mathrm{C}$ for $12 \mathrm{~min}$. Impedance changes were monitored and the area under the curve along with the amplitude and slope was calculated using the Aggrolink program (Aggrolink 5.2.3, Chrono-log, Havertown, PA, USA). Amplitude was expressed as the maximum range of platelet aggregation and the rate of the reaction was determined by drawing tangents through the steepest part of the curve. Area under the ROC (receiver operating characteristics) curve (AUC) was calculated according to the platelet aggregation curve. ${ }^{26}$ Platelet aggregation was calculated according to the following equation: (AUC 
of samples/AUC of DMSO) $\times 100 .^{28}$ Aspirin was used as a positive control for anti-platelet aggregation, as it inhibits COX-1 and reduces thromboxane A2 at low concentrations $(100 \mathrm{mg} /$ day $) .{ }^{29}$

\section{Statistical Analyses}

Statistical analysis was performed using multiple analyses of variance (ANOVA) followed by Duncan's post hoc test with repeated measures. When significant main effects or an interaction among the main effects was found, specific comparisons were made using Student's paired $t$-tests. Statistical significance was represented by $p<0.05, p<0.01$ and $p<0.001$. Statistical analysis was performed using SPSS version 13.0 statistical software (SPSS Inc., Chicago, IL, USA).

\section{RESULTS AND DISCUSSION}

\section{Analysis of the Content of the Major Components of the Extracts}

Allium cepa has long been used in our diet and is known to exert various physiological effects, including triglyceride reduction, antioxidant activities and antithrombotic effects. ${ }^{30-33}$ Flavonoids, which are primarily contained in Allium cepa, include quercetin 4'-glucoside, quercetin 3,7-diglycoside, quercetin 4',7-diglycoside, 3,4'-diglycoside, quercetin, quercetin aglycone, isorhamnetin monoglycoside and kaempferol monoglycoside. Approximately $80 \%$ of these exist as quercetin diglycoside, monoglycoside and quercetin aglycone. ${ }^{34}$ The outer shell of Allium cepa consists of approximately $6.5 \%$ quercetin in terms of shell weight and the inner portion of Allium cepa possesses $0.01 \%$ quercetin relative to its weight and has been reported to possess a relatively high quercetin content on the outer shell side. ${ }^{35}$ It has also reported that a darker skin color of Allium cepa is indicative of higher quercetin content. ${ }^{36,37}$ Despite the highest quercetin content of red Allium cepa among the various species of Allium cepa, research results regarding red Allium cepa are lacking. In this study, to increase the quercetin extraction efficiency of red Allium $c e p a$, we first manufactured Red-Q and then obtained Red-BCQ through the use of microorganisms. The quercetin peak in the Red-Q was observed at $3.9 \mathrm{~min}$ and the extract possessed a quercetin content of 2.91 $\mathrm{mg} / \mathrm{g}$. The quercetin content of the Red-BCQ obtained through the use of BS3 was $21.62 \mathrm{mg} / \mathrm{g}$ of extract (Table 1). Based on these results, the quercetin content of RedBCQ was increased by approximate 7.4-fold. The RedBCQ powder, made by adding an excipient, contains a quercetin of $4 \mathrm{mg}$ per gram of powder. This is the result of the elimination of O-linked sugars such as rutin, quercitrin and quercimeritrin by enzymes secreted by the bioconversion strains that resulted in higher relative quercetin purity.

Angelica gigas Nakai is a medicinal herb that has been demonstrated to exert a variety of pharmacological effects. This herb is characterized by its purple flowers, which are distinct from those of Angelica sinensis Diels. In particular, this herb is effective for blood circulation and it also possesses vital and anti-cancer activities that are related to hematopoietic function. D/DA, the representative substances of the Angelica gigas Nakai, are materials that have been suggested to prevent dementia. ${ }^{38,39}$ According to the Korean pharmacopoeia, Angelica gigas Nakai that can be used as an herbal medicine must contain the major ingredients D/DA. Angelica gigas Nakai possesses this major ingredient and can be used as an herbal medicine. However, Angelica sinensis Diels does not contain this ingredient in any significant quantity. The Angelica gigas Nakai used in this study was extracted according to the method of Kim $e t$ al. ${ }^{40}$ to obtain D/DA. D/DA was also analyzed using HPLC and a standard calibration curve was created based on standard materials. Our results indicated that $1 \mathrm{~g}$ of Agnex contained $0.4146 \mathrm{~g}$ of $\mathrm{D}$ and $0.3659 \mathrm{~g}$ of $\mathrm{DA}$ and the purity of these compounds was confirmed to be approximately $78 \%$. The Agnex powder, which is made by adding excipient, had a D/DA content of $120 \mathrm{mg}$ per gram of powder. This was named AG. The results are shown in Table 1.

\section{Analysis of Polyphenol and Flavonoids}

Phenol compounds are widely distributed in plants and are known to exert various effects such as antioxidant

Table 1: Quercetin content of Red-Q, Red-BCQ,

Red-BCQ powder and decursin/decursinol angelate (D/DA) content in Agnex, Agnex powder.

\begin{tabular}{|c|c|}
\hline Extract & mg of quercetin \pm SD \\
\hline Red-Q & $2.91 \pm 0.02$ in $1 \mathrm{~g}$ of Red-Q \\
\hline Red-BCQ & $21.62 \pm 0.03$ in $1 \mathrm{~g}$ of Red-BCQ \\
\hline $\begin{array}{c}\text { Red-BCQ powder } \\
(\mathrm{RA})\end{array}$ & $\begin{array}{c}4.2 \pm 0.21 \text { in } 1 \mathrm{~g} \text { of Red-BCQ powder } \\
\text { (RA) }\end{array}$ \\
\hline Extract & $\begin{array}{c}\text { mg of Decursin/Decursinol angelate } \\
\pm \text { SD }\end{array}$ \\
\hline Agnex & $780.5 \pm 0.02$ in $1 \mathrm{~g}$ of Agnex \\
\hline Agnex powder (AG) & $\begin{array}{c}120.7 \pm 0.21 \text { in } 1 \mathrm{~g} \text { of Agnex powder } \\
(\mathrm{AG})\end{array}$ \\
\hline
\end{tabular}

${ }^{a} n=3$, triplicate injection

Red-Q: Crude ethanol extract obtained from red Allium cepa. Red-BCQ: Extract obtained by bioconversion of Red-Q using $\mathrm{BS}_{3}$ strains. Red-BCQ powder: Powder made by adding excipient to Red-BCQ. This was named RA. Agnex: Extract obtained from Angelica gigas Nakai. Agnex powder: Powder made by adding excipient to Agnex. This was named AG. 
effects, anti-obesity properties and anti-inflammation effects and other physiological activities. ${ }^{41-44}$

Measurements of the flavonoid and polyphenol content of each extract and mixture revealed high values in both the RA and AG mixtures. The polyphenol and flavonoid contents of each extract and mixture are indicated in Table 2. Total polyphenol contents were found to be $69.52 \mathrm{mg}$ in RA, $70.01 \mathrm{mg}$ in AG and $143.56 \mathrm{mg}$ in RAG per gram of extract. The total flavonoid contents were found to be $26.15 \mathrm{mg}$ in RA, $20.38 \mathrm{mg}$ in $\mathrm{AG}$ and 60.24 $\mathrm{mg}$ in RAG per gram of extract.

\section{Antioxidant Activities in vitro}

It was expected that these compounds would possess strong reduction abilities, including high radial scavenging activity. DPPH radical scavenging activity, one of the representative methods of evaluating antioxidant activity, was measured. Red onion is more functional than regular white onions due to their high content of physiologically active ingredients such as anthocyanin. In particular, polyphenol, which contains a hydroxyl group, possesses suitable structure for reacting with DPPH radicals. Additionally, the majority of these ingredients are located in the exterior of the onion shell. ${ }^{45}$ Oxidative stress is closely related to fibrin clot formation and inappropriate post-translational modification of fibrin plays a role in thrombotic disorders. ${ }^{46-48}$ Antioxidant activity assays revealed that the RA and AG mixture (125-1000 $\mu \mathrm{g} /$ $\mathrm{mL}$ ) possessed potent DPPH scavenging activity (27.4$121.2 \%$ for RAG). The reducing powers of the RAG were found to be stronger than those of the RB and $A G$, respectively (Figure 1). LPS is an endotoxin produced by gram-negative bacteria. This compound causes the production of substances such as various inflammatory factors (NO) and inflammatory cytokines that result in a pathological reaction and regulates the inflammatory response. NO is generated by iNOS (inducible nitric oxide synthase) in inflammatory reactions that influence vascular control and defense and it can also act as a

\begin{tabular}{|c|c|c|}
$\begin{array}{r}\text { Table 2: Polyphenol and flavonoid contents of RA, } \\
\text { AG and a mixture of these two compounds. }\end{array}$ \\
\hline \multirow{2}{*}{ Extract } & \multicolumn{2}{|c|}{ Component(mg/g) } \\
\cline { 2 - 3 } & Total polyphenol & Total flavonoid \\
\hline RA & $69.52 \pm 1.69^{* * *}$ & $26.15 \pm 0.17^{* * *}$ \\
\hline AG & $70.01 \pm 0.20^{\star * *}$ & $20.38 \pm 0.94^{* * *}$ \\
\hline RAG & $143.46 \pm 2.30^{* * *}$ & $60.24 \pm 5.24^{* * *}$ \\
\hline
\end{tabular}

RA: Red-BCO powder, AG: Agnex powder, RAG: Mixture of RA+AG (RA: AG = 2:1). Data are means $\pm S D$ of triplicate determinations. Different superscripts within a column indicate statistically significant differences $\left({ }^{* * *} p<0.001\right)$. neurotransmitter under normal conditions. However, excessive NO production activates macrophages by producing harmful substances such as peroxynitrite and nitrogen dioxide, both of which act as inflammatory stimulants. ${ }^{49}$ In this study, RAW 264.7 cells were treated with LPS to induce inflammation and then RA, AG, or a mixture of these two compounds was added to observe the inhibitory effect on NO generation. All substances inhibited NO production at a level similar to the control (ascorbic acid) and there were no significant differences in concentration. LPS was treated in RAW264.7 cells to induce inflammation and add various concentrations of samples to observe NO production inhibitory effect. All samples inhibited NO production to a level similar to the control ascorbic acid. Potent nitrite scavenging activity was observed for RA, AG and RAG (Figure 2).

\section{Anticoagulant Activity in vitro}

Wang et al. suggested that oxidation stress increases the modification of fibrin and RBC hemolysis. ${ }^{46-48}$ Thus, the strong antioxidant action of these extracts may delay blood coagulation formation. Based on the knowledge that excessive blood coagulation can be harmful to the regulation of homeostasis, the significant effects of RA, AG and RAG on coagulation factors as measured by PT and aPTT were investigated. The effects of RA, AG and RAG on coagulation parameters (aPTT, TT and PT) are shown in Table 3. In this study, RA and AG exhibited beneficial effects on external coagulation pathways by significantly expanding aPT'T and these compounds may interfere with fibrin formation. However, a combination of RA and AG is considered to have a weak synergy effect. The anti-coagulation activities of the samples were measured by assessing TT, PT and aPTT. Treatment with aspirin $(1.5 \mathrm{mg} / \mathrm{mL})$ as a positive control extended

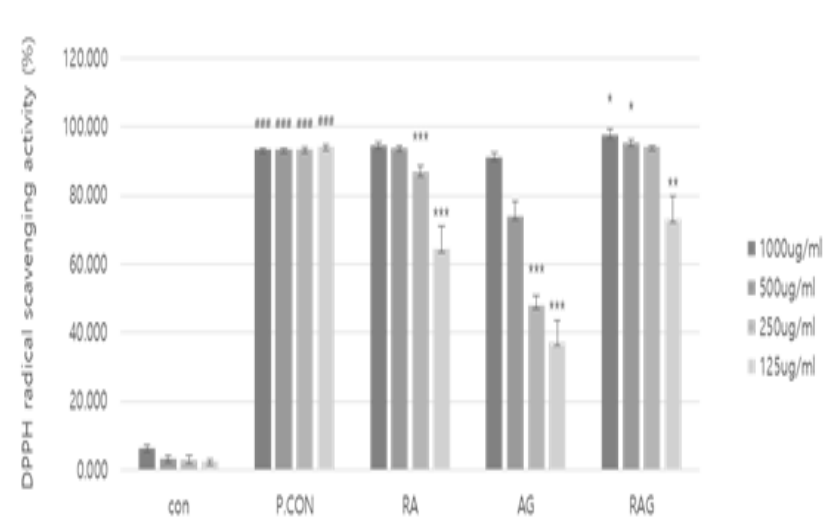

Figure 1: Effects of RA, AG and a mixture of these compounds on DPPH radical scavenging activity.

Con: Phosphate Buffered Saline (PBS), P.CON: Ascorbic acid as positive control, RA: Red-BCQ powder, AG: Agnex powder, RAG: Mixture of RA and AG (RA:AG = 2:1). All values were expressed as means \pm SD. Significance differences from control value are indicated by $* p<0.5, * * p<0.01$ and $* * * p<0.001$. 
Table 3: Effect of the RA, AG and their mixture on blood coagulation.

\begin{tabular}{|c|c|c|c|c|}
\hline \multirow{2}{*}{$\begin{array}{c}\text { Extract/ } \\
\text { Chemicals }\end{array}$} & $\begin{array}{c}\text { Concentration } \\
(\mathbf{m g} / \mathbf{m l})\end{array}$ & \multicolumn{3}{|c|}{ Anticoagulation Activity (Multiplication of Control) } \\
\cline { 3 - 5 } & - & TT & PT & aPTT \\
\hline DMSO & 1.5 & $1.00 \pm 0.02$ & $1.00 \pm 0.01$ & $1.00 \pm 0.00$ \\
\hline Aspirin & 5 & $>15.0$ & $>15.0$ & $>15.0$ \\
\hline Aspirin & 5 & $1.01 \pm 0.03^{* *}$ & $1.29 \pm 0.00$ & $1.39 \pm 0.13$ \\
\hline RA & 5 & $0.97 \pm 0.08^{* *}$ & $1.23 \pm 0.19$ & $1.23 \pm 0.23$ \\
\hline AG & 5 & $0.99 \pm 0.08^{* *}$ & $1.02 \pm 0.00^{* *}$ & $1.37 \pm 0.02^{*}$ \\
\hline RAG & & &
\end{tabular}

Anti-coagulation activity was calculated using the clotting time of a given sample divided by the clotting time of the solvent control in blood coagulation assays.

TT: thrombin, PT: prothrombin, aPTT: activated partial thromboplastin times

Aspirin: Positive Control, RA: Red-BCO powder, AG: Agnex powder, RAG: Mixture of RA and AG (RA: AG = 2:1). Data are means $\pm S D$ of triplicate determinations. Different superscripts within a column indicate statistically significant differences $\left({ }^{* *} p<0.005,{ }^{*} p<0.05\right)$.

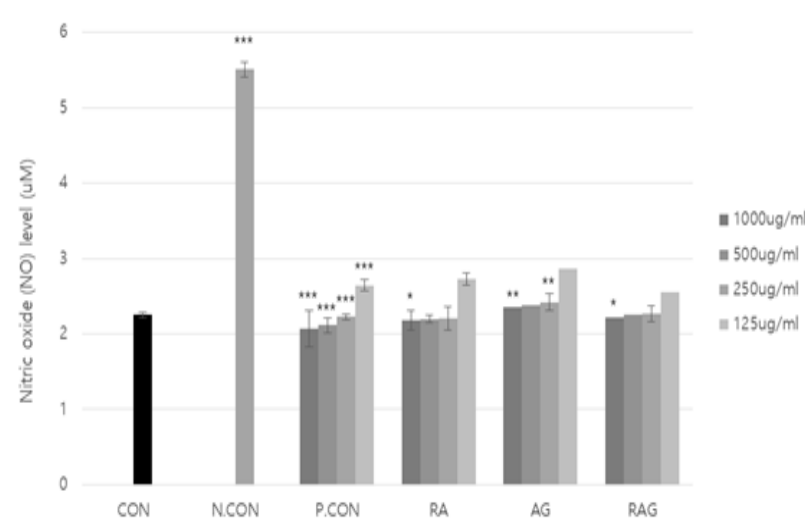

Figure 2: Effects of RA, AG and a mixture of these compounds on nitric oxide production in Raw 264.7 cells treated with LPS. NO concentration was determined using the Griess reaction. Con: Phosphate Buffered Saline (PBS), P.CON: Ascorbic acid as positive control, RA: Red-BCQ powder, AG: Agnex powder, RAG: Mixture of RA and AG (RA: AG = 2:1). All values were expressed as means \pm SD. Significance differences from control value are indicated by ${ }^{*} p<0.5,{ }^{* *} p<0.01$ and ${ }^{* * *} p<0.001$.

the coagulation time to 1.83-fold for TT, 1.29-fold for PT and 1.48-fold for aPT'T compared to the coagulation times of non-treatment samples (DMSO as solvent control) (Table 3). Treatment with high-concentrations of aspirin $(5 \mathrm{mg} / \mathrm{mL})$ extended the clotting time to greater than 15-fold for TT, PT and aPTT compared to clotting times observed with non-treatment. Treatment with RA $(5.0 \mathrm{mg} / \mathrm{mL})$ was found to significantly extend PT (1.29-fold) and aPTT (1.39-fold). AG (5.0 mg/mL) treatment extended the coagulation time by 1.23 -fold for PT and 1.23-fold for aPTT. However, combined treatment with RA and AG showed weak synergy effect (Table 3).

\section{Platelet Aggregation Activities in vitro}

RA alone promoted platelet aggregation according to our results (Table 4). However, our results regarding

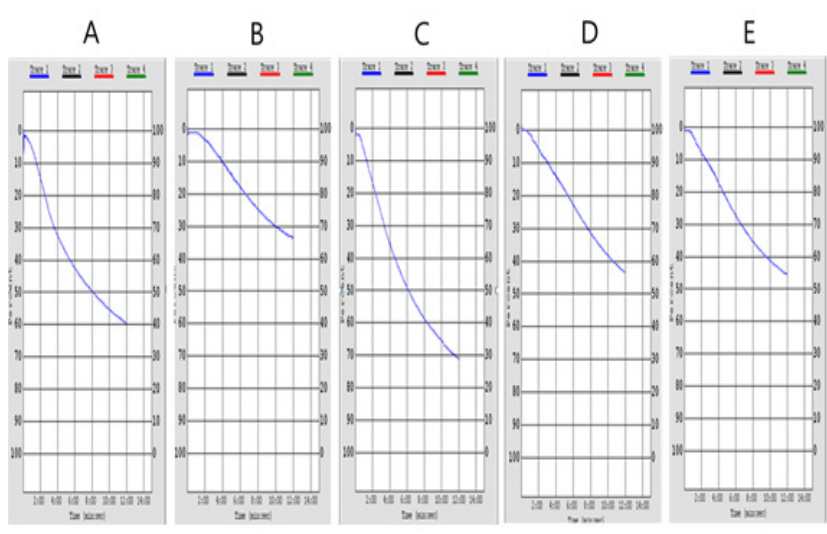

Figure 3: Impedance changes during platelet aggregation after the addition of aspirin and the RA and AG into a whole blood aggregometer.

(A) DMSO, (B) aspirin $(250 \mu \mathrm{g} / \mathrm{ml})$, (C) RA (Red-BCQ powder, $250 \mu \mathrm{g} / \mathrm{ml})$, (D) AG (Agnex powder, $250 \mu \mathrm{g} / \mathrm{ml}$ ), (E) RAG (RA: AG $=2: 1,250 \mu \mathrm{g} / \mathrm{ml}$ ), respectively.

platelet aggregation inhibitory effects did reveal that they were increased after AG treatment alone or after a combined treatment with RA and AG. Further research will be needed to clarify the underlying mechanism. Based on the observation that platelet aggregation is strongly inhibited by aspirin, the changes in PAR (platelet aggregation ratio) in response to aspirin treatment were determined. At $250 \mu \mathrm{g} / \mathrm{ml}$ of aspirin, the PARs were $44.32 \%$ compared to those observed after non-treatment (Table 4, Figure 3). Strong and rapid activations of platelet aggregation were identified in response to treatment with $\mathrm{RA}(250 \mu \mathrm{g} / \mathrm{ml})$. The PARs of $A G$ and RAG were $62.63 \%$ and $68.36 \%$, respectively, compared to those of the non-treatment samples. RA treatment $(250 \mu \mathrm{g} / \mathrm{ml})$ failed to alter platelet aggregation. Therefore, when AG is subjected to combined treatment, the platelet aggregation inhibitory effects are facilitated by the action of the AG. 
Table 4: Effect of the RA, AG and their mixture on platelet aggregation.

\begin{tabular}{|c|c|c|c|c|c|c|}
\hline $\begin{array}{c}\text { Samples } / \\
\text { Chemicals }\end{array}$ & $\begin{array}{c}\text { Concentration } \\
(\boldsymbol{\mu} \mathbf{g} / \mathbf{m l})\end{array}$ & $\begin{array}{c}\text { Amplitude } \\
(\mathbf{o h m})\end{array}$ & Slope & $\begin{array}{c}\text { Lag } \\
\text { time } \\
(\mathbf{s e c})\end{array}$ & $\begin{array}{c}\text { Area } \\
\text { under }\end{array}$ & $\begin{array}{c}\text { PAR } \\
(\%)\end{array}$ \\
\hline DMSO & - & 14 & 3 & 36 & 106.5 & 100.00 \\
\hline Aspirin & 250 & 8 & 1 & 128 & 47.2 & 44.32 \\
\hline RA & 250 & 18 & 2 & 30 & 125.1 & 117.46 \\
\hline AG & 250 & 11 & 1 & 56 & 66.7 & 62.63 \\
\hline RAG & 250 & 11 & 2 & 48 & 72.8 & 68.36 \\
\hline
\end{tabular}

PAR: Platelet Aggregation Ratio. Data are presented as representative results relative to three independent determinations. Amplitude is expressed as ohms by maximum extent of platelet aggregation and slope (rate of reaction) is determined by drawing a tangent through the steepest part of curve. Area under the curve (AUC) was calculated from the platelet aggregation curve. Aspirin: Positive Control, RA: Red-BCQ powder, AG: Agnex powder, RAG: Mixture of RA and AG (RA: AG = 2:1).

\section{CONCLUSION}

We examined the effects of Red Allium cepa and Angelica gigas Nakai as food sources containing quercetin and D/ DA on blood coagulation and thrombosis. Analyses of anti-coagulation, platelet aggregation and antioxidant activity of RA and AG revealed that these extracts possess distinct biological functionalities. The flavonoid and polyphenol content was higher in the mixture than it was in either RA or AG individually. In agreement with this, DPPH scavenging activity and nitrate scavenging activity were also both higher in the RAG mixture group. Cell viability assays also revealed no cytotoxicity at high concentrations $(1000 \mu \mathrm{g} / \mathrm{ml})$. RA and AG each possess distinct anti-coagulation activities. However, we confirmed that these compounds have weak synergy effect. Although the antiplatelet activity was strong for AG, there was no antiplatelet activity exerted by RA. Based on these results, we are currently conducting in vivo test and will publish a follow-up paper through further verification in the future.

\section{ACKNOWLEDGEMENT}

This work was supported by the Korea Institute of Planning and Evaluation for Technology in Food, Agriculture and Forestry (IPET) through the High Value-added Food Technology Development Program (Grant Number 317067-3).

\section{CONFLICT OF INTEREST}

The authors declare that there is no conflict of interest.

\section{ABBREVIATIONS}

Agnex: Angelica gigas Nakai extract; Red-Q: Ethanol extract of red Allium cepa; Red-BCQ: Bioconversioned quercetin of Red-Q; RA: Red-BCQ powder; AG: Agnex powder; RAG: Mixture of RA and AG (RA:AG = 2:1); DMSO: dimethyl sulfoxide; DPPH: 1,1-diphenyl-2picryl hydrazyl; NO: Nitric oxide; iNOS: inducible nitric oxide synthase; TT: thrombin time; PT: Prothrombin time; aPTT: active partial thromboplastin time; PAR: Platelet Aggregation Ratio; AUC: Area under the curve.

\section{REFERENCES}

1. Lachman J, Pronek D, Hejtmánková A, Dudjak J, Pivec V, Faitová K. Total polyphenol and main flavonoid antioxidants in different onion (Allium cepa L.) varieties. Hortic Sci. 2003;30(4):142-7.

2. Hossain MB, Camphuis G, Aguiló-Agayo I, Gangopadhyay N, Rai DK. Antioxidant activity guided separation of major polyphenols of marjoram (Origanum majorana L.) using flash chromatography and their identification by liquid chromatography coupled with electrospray ionization tandem mass spectrometry. J Sep Sci. 2014;37(22):3205-13.

3. Dini I, Tenore GC, Dini A. Chemical composition, nutritional value and antioxidant properties of Allium cepa L. Var. tropeana (red onion) seeds. Food Chem. 2008;107(2):613-21.

4. Roldán-Marín E, Krath BN, Poulsen M, Binderup ML, Nielsen TH, Hansen $\mathrm{M}$, et al. Effects of an onion by-product on bioactivity and safety markers in healthy rats. Br J Nutr. 2009;102(11):1574-82.

5. Park J, Kim J, Kim MK. Onion Flesh and Onion Peel Enhance Antioxidant Status in Aged Rats. J Nutr Sci Vitaminol. 2007;53(1):21-9.

6. Singh BN, Singh BR, Singh RL, Prakash D, Singh DP, Sarma BK, et al. Polyphenolics from various extracts/fractions of red onion (Allium cepa.) peel with potent antioxidant and antimutagenic activities. Food Chem Toxicol. 2009;47(6):1161-7.

7. Murota K, Hotta A, Ido H, Kawai $\mathrm{Y}$, Moon JH, Sekido K, et al. Antioxidant capacity of albumin-bound quercetin metabolites after onion consumption in humans. J Med Invest. 2007;54(3,4):370-4.

8. Huang D, Ou B, Prior RL. The chemistry behind antioxidant capacity assays. J Agric Food Chem. 2005;53(6):1841-56.

9. Ahn KS, Sim WS, Kim IH. Decursin: A cytotoxic agent and protein kinase C activator from the root of Angelica gigas. Planta Med. 1996;62(01):7-9.

10. Bae EA, Han MJ, Kim NJ, Kim DH. Anti-Helicobacter pylori activity of herbal medicines. Biol Pharm Bull. 1998;21(9):990-2.

11. Kang SY, Lee KY, Sung SH, Park MJ, Kim YC. Coumarins isolated from Angelica gigas inhibit acetylcholinesterase structure-activity relationships. J Nat Prod. 2001;64(5):683-5.

12. Lee SH, Lee YS, Jung SH, Shin KH, Kim BK, Kang SS. Anti-tumor activities of decursinol angelate and decursin from Angelica gigas. Arch Pharm Res. 2003;26(9):727-30.

13. Lee SH, Shin DS, Kim JS, Oh KB, Kang SS. Antibacterial coumarins from Angelica gigas roots. Arch Pharm Res. 2003;26(6):449-52. 
14. Yim DS, Singh RP, Agarwal C, Lee SY, Chi HJ, Agarwal R. A novel anticancer agent, decursin, induces $\mathrm{G} 1$ arrest and apoptosis in human prostate carcinoma cells. Cancer Res. 2005;65(3):1035-44.

15. Kim KM, Jung JY, Hwang SW, Kim MJ, Kang JS. Isolation and purification of decursin and decursinol angelate in Angelica gigas Nakai. J Kor Soc Food Sci Nutr. 2009;38(5):653-6.

16. Kim GS, Park CG, Jeong TS, Cha SW, Baek NI, Song KS. ACAT (AcylCoA: cholesterol Acyltransferase) inhibitory effect and quantification of pyranocoumarin in different parts of Angelica gigas Nakai. J Appl Biol Chem. 2009;52(4):188-94.

17. Kim MS, Kang DK, Shin WC, Sohn HY. Anti-microbial, anti-oxidant and antithrombosis activity of lees of bokbunjawine (RubuscoreanusMiquel). J Life Sci. 2015;25(7):757-64.

18. Ku SK, Bae JS. Antiplatelet and antithrombotic activities of purourogallin in vitro and in vivo. BMB Rep. 2014;47(7):376-81.

19. Berry CN, Girard D, Lochot S, Lecoffre C. Antithrombotic actions of argatroban in rat models of venous, 'mixed' and arterial thrombosis and its effects on the tail transection bleeding time. British J Pharmacol. 1994;113(4):1209-14.

20. Monagle P. Thrombosis congenital heart disease and thrombosis: What do we know?. Nat Rev Cardiol. 2014;11(3):132-4.

21. Kattula S, Byrnes JR, Wolberg AS. Fibrinogen and fibrin in hemostasis and thrombosis. Arterioscler Thromb Vasc Biol. 2017;37(3):e13-21.

22. Folin O, Denis W. A colorimetric method for the determination of phenols (and phenolderivatives) in urine. J Biol Chem. 1915;22(2):305-8.

23. Davis WB. Determination of flavonones in citrus fruits. Anal Chem. 1947;19(7):476-8.

24. Blois MS. Antioxidant determination by the use of a stable free radical. Nature. 1958;181(4617):1199-200.

25. Granger DL, Taintor RR, Boockvar KS, Hibbs JB. Measurement of nitrate and nitrite in biological samples using nitrate reductase and Griess reaction. Methods Enzymol. 1996;268:142-51.

26. Kim MS, Shin WC, Kang DK, Sohn HY. Anti-thrombosis activity of sinapic acid isolated from the lees of bokbunja wine. J Microbiol Biotechnol. 2016;26(1):61-5.

27. Szczeklik A, Musial J, Undas A, Swadzba J, Gora PF, Piwowarska W, et al. Inhibition of thrombin generation by aspirin is blunted in hypercholesterolemia. Arterioscler. Thromb Vasc Biol. 1996;16(8):948-54.

28. Sweeney JD, Hoerning LA, Behrens AN, Novak E, Swank RT. Von willebrand's variant (type II buffalo.) Thrombocytopenia after desmopressin but absence of in vitro hypersensitivity to ristocetin. Am J Clin Path. 1990;93(4):522-5.

29. Wang J, Xiong $X$, Feng B. Aspirin resistance and promoting blood circulation and removing blood stasis: Current situation and prospectives. Evid Complement Alter Med. 2014;7330:1-11.

30. Bakhsh R, Khan S. Influence of onion (Allium cepa) and chaunga (Caraluma tubercula) on serum cholesterol, triglycerides, total lipids in human subject. Sarhad J Agric. 1990;6:425-8.

31. Kendler BS. Garlic (Allium sativum) and onion (Allium cepa): A review of their relationship to cardiovascular disease. Prev Med. 1987;16(5):670-85.

32. Kim SK, Kim MK. Effect of dried powders or ethanol extracts of onion flesh and peel on lipid metabolism, antioxidative and antithrombogenic capacities in 16-month- old rats. Kor J Nutr. 2004;37(8):623-32.
33. Shon MY, Choi SD, Kahng GG, Nam SH, Sung NJ. Antimutagenic, antioxidant and free radical scavenging activity of ethyl acetate extracts from white, yellow and red onions. Food Chem Toxicol. 2004;42(4):659-66.

34. Lu XL, Zhao $\mathrm{CH}$, Yao XL, Zhang $\mathrm{H}$. Quercetin attenuates high fructose feeding-induced atherosclerosis by suppressing inflammation and apoptosis via ROS-regulated PI3K/AKT signaling pathway. Biomed Pharmacother. 2017;85:658-71.

35. Kwak JH, Seo JM, Kim NH, Arasu MV, Kim S, Yoon MK, et al. Variation of quercetin glycoside derivatives in three onion (Allium cepa L.) varieties. Saudi J Biol Sci. 2017;24(6):1387-91.

36. Hermann K. Flavonols and flavones in food plants: A review. J Food Tech. 1976;11(5):433-88.

37. Kwak JH, Seo JM, Kim NH, Arasu MV, Kim SG, Yoon MK, et al. Variation of quercetin glycoside derivatives $\mathrm{n}$ three onion (Allium cepa L.) varieties. Saudi J Biol Sci. 2017;24(6):1387-91.

38. Pearson RCA, Sofroniew MV, Cuello AC, Powell TPS, Eckenstein F, Esiri MM, et al. Persistence of cholinergic neurons in the basal nucleus in a brain with senile dementia of the Alzheimer's type demonstrated by Immunohistochemical staining for choline acetyltransferase. Brain Res.1983;289(1-2):375-9.

39. Li L, Li W, Jung SW, Lee YW, Kim YH. Protective effects of decursin and decursinol angelate against amyloid $\beta$-protein-induced oxidative stress in the PC12 cell line: The role of Nrf2 and antioxidant enzymes. Biosci Biotechnol Biochem. 2011;75(3)434-42.

40. Kim KM, Jung JY, Hwang SW, Kim MJ, Kang JS. Isolation and Purification of decursin and decursinol Angelate in Angelica gigas Nakai. J Kor Soc Food Sci Nutr. 2009;38(5):653-6.

41. Lee HJ, Lee KH, Park EJ, Jung HK. Effect of onion extracts on serum cholesterol in borderline hypercholesterolemic participants. J Kor Soc Food Sci Nutr. 2010;39(12):1783-9.

42. Lee LS, Choi EJ, Kim CH, Kim YB, Kum JS, Park JD. Quality characteristics and antioxidant properties of black and yellow soybeans. Kor J Food Sci Technol. 2014;46(6):757-61.

43. Rha YA, Choi MS, Park SJ. Antioxidant and anti-adipogenic effects of fermented Rhusverniciflua. Kor J Culinary Res. 2014;20(3):137-47.

44. Rice-Evans C, Miller N, Paganga G. Antioxidant properties of phenolic compounds. Trends Plant Sci. 1997;2(4):152-9.

45. Heo JS, Cha JY, Kim HW, Ahn HY, Eom KE, Heo SJ, et al. Bioactive materials and biological activity in the extracts of leaf, stem mixture and root from Angelica gigas Nakai. Kor J Life Sci. 2010;20(5):750-9.

46. Wang L, Li L, Wang H, Liu J. Study on the influence of oxidative stress on the fibrillization of fibrinogen. Biochem J. 2016;473(23):4373-84.

47. Martinez M, Weisel JW, Ischiropoulos H. Functional impact of oxidative posttranslational modifications on fibrinogen and fibrin clots. Free Radic Biol Med. 2013;65:411-8.

48. Bijak M, Nowak P, Borowiecka M, Ponczek MB, Zbikowska HM, Wachowicz B. Protective effects of (-)-epicatechin against nitrative modifications of fibrinogen. Thromb Res. 2012;130(3):e123-8.

49. Ippouchi $\mathrm{K}$, Itou $\mathrm{H}$, Azuma $\mathrm{K}$, Higashio $\mathrm{H}$. Effect of naturally occurring organosulfur compounds on nitric oxide production in lipopolysaccharideactivated macrophages. Life Sci. 2002;71(4):411-9. 


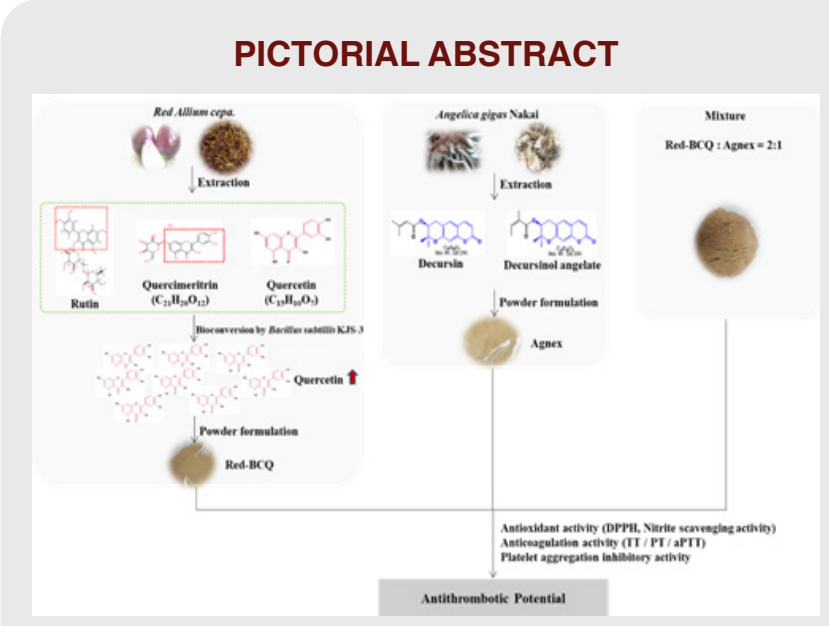

\section{SUMMARY}

We investigated the effects of red Allium cepa (RA) and Angelica gigas Nakai (AG) as food sources containing quercetin and D/DA on blood coagulation and thrombosis. The analysis of anti-coagulation, platelet aggregation and antioxidant activity of RA and AG showed that these extracts had distinct biological functionalities. RA and AG each have distinct anticoagulation activities. Although the antiplatelet activity was strong for $A G$, there was weak antiplatelet activity exerted by RA. Therefore, mixture of RA and AG will have anti-coagulation activity and anti-platelet activity. Based on these results, we are currently conducting in vivo test and will publish a follow-up paper through further verification in the future.

\section{About Authors}

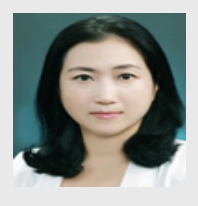

Prof. Jin Young Lee, Department of Pharmacy, Kyungsung University, 309, Suyeong-ro, Nam-gu, Busan, Korea.

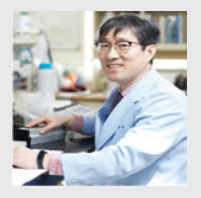

Prof. Jae Seon Kang, Dean of the College of Pharmacy, Department of Pharmacy, Kyungsung University, 309, Suyeong-ro, Nam-gu, Busan, Korea.

Cite this article: Lee JY, Kang JS. Antithrombotic Potential of Red Allium cepa and Angelica gigas Nakai. Indian J of Pharmaceutical Education and Research. 2021;55(3):837-45. 